\title{
Optical Anisotropy of Zinc-Blende Semiconductors in an Electric Field
}

\author{
Bradley A. Foreman \\ Department of Physics, Hong Kong University of Science and Technology, Clear Water Bay, Kowloon, Hong Kong, China
}

(Received 5 November 1999)

A theory of optical anisotropy for zinc-blende semiconductors in an electric field is derived by extending the formalism of Luttinger and Kohn [Phys. Rev. 97, 869 (1955)] to higher order. This resolves a recent controversy over the correct form of the Hamiltonian for the degenerate valence bands.

PACS numbers: 78.20.Jq, 71.20.Nr, 78.20.Bh, 78.40.Fy

One of the outstanding problems in solid state physics has been that of a periodic crystal in a uniform electric field. The presence of a field gives rise to the possibility of such interesting phenomena as Bloch current oscillations, Wannier-Stark quantization of the energy levels, Zener tunneling between bands, and Franz-Keldysh oscillations in the absorption spectrum. Recently, Khurgin and Voisin [1] have drawn attention to a fundamental problem in this area that is still unsolved-namely, how to describe the influence of an electric field on degenerate Bloch bands, such as the $\Gamma_{15}$ valence bands in a zinc-blende semiconductor.

Interest in this topic has been aroused by the discovery of the quantum-well Pockels effect [2-4], in which the optical properties (absorption and permittivity) of a quantum well in an electric field exhibit an unusually large in-plane polarization anisotropy. Although the anisotropy due to the broken crystal symmetry at a heterojunction is now reasonably well understood $[5,6]$, the contribution from the field in bulk material remains controversial [1,7].

It is somewhat surprising that such a basic problem was not solved in the 1950's and 1960's, when effectiveHamiltonian theory and the Franz-Keldysh effect [8-10] were under heavy investigation. The reason for this lies in the fact that the most obvious approach to the problemsolving the Schrödinger equation in the Bloch representation $[11,12]-$ is intractable. As noted by Wannier [13], the coordinate operator in the Bloch representation is a singular function of $\mathbf{k}$ at a degeneracy [12, p. 331]. To avoid this difficulty, previous analyses of Franz-Keldysh electroabsorption have used a simplified two-band model with no degeneracy [8-10]. However, such a model does not give rise to optical anisotropy.

An alternative approach, suggested by Wannier [13] but never implemented, is to use the Luttinger-Kohn representation [14], in which the crystal Hamiltonian is not diagonal, but the coordinate operator is an analytic function of $\mathbf{k}$. This representation is thus well suited for the treatment of degenerate bands. The paper of Luttinger and Kohn treats explicitly only the lowest-order effective-mass approximation, but there is no difficulty in principle in extending their analysis to higher order. The present work takes this approach, with the goal of (i) establishing a valid Hamiltonian for degenerate bands in an electric field and (ii) using this theory to calculate the anisotropy in the near-band-gap absorption of a zinc-blende semiconductor in an electric field.

The results derived in this Letter show unequivocally that the mechanisms for bulk anisotropy proposed in several recent articles $[1,3,15]$ are incorrect. It is shown here that in the Luttinger-Kohn representation the two main linear-field mechanisms are (i) polarization of the electron wave function, which changes the interband momentum matrix elements but does not split the valence-band states at $\mathbf{k}=\mathbf{0}$ and (ii) piezoelectric-induced splitting of the valence bands.

The Luttinger-Kohn representation uses basis states $|n \mathbf{k}\rangle$, where $n$ labels the zone-center Bloch functions $u_{n 0}(\mathbf{x})$, and $\mathbf{k}$ is a wave vector in the first Brillouin zone. In coordinate space, the basis functions are $\langle\mathbf{x} \mid n \mathbf{k}\rangle=$ $e^{i \mathbf{k} \cdot \mathbf{x}} u_{n 0}(\mathbf{x})$. Every operator considered below has matrix elements of the form $\left\langle n \mathbf{k}|A| n^{\prime} \mathbf{k}^{\prime}\right\rangle=\left\langle n|A| n^{\prime}\right\rangle \delta\left(\mathbf{k}-\mathbf{k}^{\prime}\right)$, where $\left\langle n|A| n^{\prime}\right\rangle$ is an operator [16]. Thus, for brevity I shall give only $\left\langle n|A| n^{\prime}\right\rangle$.

The Hamiltonian is $H=H_{0}+U$, where $H_{0}=H^{(0)}+$ $H^{(1)}$ is periodic, with matrix elements [14]

$$
\begin{gathered}
\left\langle n\left|H^{(0)}\right| n^{\prime}\right\rangle=\left(\epsilon_{n}+\frac{\hbar^{2} k^{2}}{2 m}\right) \delta_{n n^{\prime}}, \\
\left\langle n\left|H^{(1)}\right| n^{\prime}\right\rangle=\frac{\hbar}{m} k_{\alpha} p_{n n^{\prime}}^{\alpha} .
\end{gathered}
$$

Here $\epsilon_{n}$ is the energy of state $u_{n 0}, m$ is the mass of a free electron, and $p_{n n^{\prime}}^{\alpha}$ is the $\alpha$ component of the momentum matrix element between $u_{n 0}$ and $u_{n^{\prime} 0}$ (a sum on $\alpha$ is implied). For a uniform electric field $\mathbf{E}$, the potential energy $U$ has the form $U(\mathbf{x})=e \mathbf{E} \cdot \mathbf{x}(e>0)$, so its matrix elements are given by Eq. (II.44) of Ref. [14]:

$$
\left\langle n|U| n^{\prime}\right\rangle=i e \mathbf{E} \cdot \nabla_{\mathbf{k}} \delta_{n n^{\prime}} .
$$

The goal is to find a basis in which $H$ has no interband matrix elements to first order in $k$ and $E$; states with different $\epsilon_{n}$ are thereby decoupled to second order in $k$ and $E$. One starts by eliminating the $\mathbf{k} \cdot \mathbf{p}$ coupling $H^{(1)}$. This is done by applying a unitary transformation $\bar{H}=e^{-S} H e^{S}=H+[H, S]+\frac{1}{2}[[H, S], S]+\ldots$, in which $S$ is chosen such that $H^{(1)}+\left[H^{(0)}, S\right]=0$ [14]:

$$
\begin{aligned}
\left\langle n|S| n^{\prime}\right\rangle & =-\frac{k_{\alpha} p_{n n^{\prime}}^{\alpha}}{m \omega_{n n^{\prime}}} \quad \text { if } \omega_{n n^{\prime}} \neq 0, \\
& =0 \quad \text { if } \omega_{n n^{\prime}}=0,
\end{aligned}
$$


where $\hbar \omega_{n n^{\prime}}=\epsilon_{n}-\epsilon_{n^{\prime}}$. (Note that in a zinc-blende crystal, $p_{n n^{\prime}}^{\alpha}=0$ whenever $\omega_{n n^{\prime}}=0$ [17].) With this choice, the Hamiltonian $\bar{H}$ is

$$
\bar{H}=H^{(0)}+\frac{1}{2}\left[H^{(1)}, S\right]+U+[U, S]+\frac{1}{2}[[U, S], S],
$$

which is valid to second order (thus omitting terms of order $\left.k^{2} E\right)$. The term $\frac{1}{2}\left[H^{(1)}, S\right]$ is just the usual effective-mass correction,

$$
\frac{1}{2}\left\langle n\left|\left[H^{(1)}, S\right]\right| n^{\prime}\right\rangle=\frac{\hbar k_{\alpha} k_{\beta}}{2 m^{2}} \sum_{i} p_{n i}^{\alpha} p_{i n^{\prime}}^{\beta}\left(\omega_{n i}^{-1}+\omega_{n^{\prime} i}^{-1}\right) .
$$

In conventional effective-mass theory, the terms $[U, S]$ and $\frac{1}{2}[[U, S], S]$ are omitted [14]. Here they are retained, since they represent the lowest-order band-mixing effects arising from an electric field. From (3) and (4), one has

$$
\begin{gathered}
\left\langle n|[U, S]| n^{\prime}\right\rangle=-\frac{i e E_{\alpha} p_{n n^{\prime}}^{\alpha}}{m \omega_{n n^{\prime}}} \text { if } \omega_{n n^{\prime}} \neq 0, \\
=0 \quad \text { if } \omega_{n n^{\prime}}=0, \\
\frac{1}{2}\left\langle n|[[U, S], S]| n^{\prime}\right\rangle=\frac{i e}{2 m^{2}}\left(E_{\alpha} k_{\beta}-k_{\alpha} E_{\beta}\right) \\
\times \sum_{i} \frac{p_{n i}^{\alpha} p_{i n^{\prime}}^{\beta}}{\omega_{n i} \omega_{i n^{\prime}}} .
\end{gathered}
$$

The next task is to eliminate the first-order interband terms in Eq. (7). This is done by applying a second unitary transformation $\hat{H}=e^{-R} \bar{H} e^{R}$, in which $R$ is chosen such that $[U, S]+\left[H^{(0)}, R\right]=0[18]$ :

$$
\begin{aligned}
\left\langle n|R| n^{\prime}\right\rangle & =\frac{i e E_{\alpha} p_{n n^{\prime}}^{\alpha}}{\hbar m \omega_{n n^{\prime}}^{2}} \text { if } \omega_{n n^{\prime}} \neq 0, \\
& =0 \text { if } \omega_{n n^{\prime}}=0 .
\end{aligned}
$$

The new Hamiltonian is therefore

$$
\begin{aligned}
\hat{H}= & H^{(0)}+\frac{1}{2}\left[H^{(1)}, S\right]+U+\frac{1}{2}[[U, S], S] \\
& +\frac{1}{2}[[U, S], R],
\end{aligned}
$$

where the identity $[U, R]=0$ has been used. The only undetermined quantity in $(10)$ is $\frac{1}{2}[[U, S], R]$ :

$$
\begin{aligned}
\frac{1}{2}\left\langle n|[[U, S], R]| n^{\prime}\right\rangle= & -\frac{e^{2} E_{\alpha} E_{\beta}}{2 \hbar m^{2}} \\
& \times \sum_{i} \frac{p_{n i}^{\alpha} p_{i n^{\prime}}^{\beta}}{\omega_{n i} \omega_{i n^{\prime}}}\left(\omega_{n i}^{-1}+\omega_{n^{\prime} i}^{-1}\right) .
\end{aligned}
$$

Equation (10) achieves the desired second-order decoupling of the energy bands.

To calculate optical transition rates, one also needs to know the momentum operator. This is obtained from the unitary transformations $\overline{\mathbf{p}}=e^{-S} \mathbf{p} e^{S}$ and $\hat{\mathbf{p}}=e^{-R} \overline{\mathbf{p}} e^{R}$; to first order in $k$ and $E$, one has

$$
\hat{\mathbf{p}}=\mathbf{p}+[\mathbf{p}, S]+[\mathbf{p}, R] .
$$

The matrix elements of these operators are

$$
\begin{gathered}
\left\langle n\left|p^{\alpha}\right| n^{\prime}\right\rangle=p_{n n^{\prime}}^{\alpha}+\hbar k_{\alpha} \delta_{n n^{\prime}}, \\
\left\langle n\left|\left[p^{\alpha}, S\right]\right| n^{\prime}\right\rangle=\frac{k_{\beta}}{m} \sum_{i}\left(\frac{p_{n i}^{\alpha} p_{i n^{\prime}}^{\beta}}{\omega_{n^{\prime} i}}+\frac{p_{n i}^{\beta} p_{i n^{\prime}}^{\alpha}}{\omega_{n i}}\right), \\
\left\langle n\left|\left[p^{\alpha}, R\right]\right| n^{\prime}\right\rangle=\frac{i e E_{\beta}}{\hbar m} \sum_{i}\left(\frac{p_{n i}^{\alpha} p_{i n^{\prime}}^{\beta}}{\omega_{n^{\prime} i}^{2}}-\frac{p_{n i}^{\beta} p_{i n^{\prime}}^{\alpha}}{\omega_{n i}^{2}}\right) .
\end{gathered}
$$

The states of interest are the $\Gamma_{1}$ conduction $(n=S)$ and $\Gamma_{15}$ valence $\left(n^{\prime}=X, Y\right.$, or $\left.Z\right)$ bands of a zinc-blende semiconductor. The symmetry properties of these states $[17,19]$ can be used to write $\langle S|\hat{\mathbf{p}}| X\rangle$ as

$$
\begin{aligned}
\frac{\hbar}{i m}\langle S|\hat{\mathbf{p}}| X\rangle= & \hat{\mathbf{x}} P+\hat{\mathbf{y}}\left(D E_{z}-i B k_{z}\right) \\
& +\hat{\mathbf{z}}\left(D E_{y}-i B k_{y}\right),
\end{aligned}
$$

with $\langle S|\hat{\mathbf{p}}| Y\rangle$ and $\langle S|\hat{\mathbf{p}}| Z\rangle$ obtained from cyclic permutations of $x, y$, and $z$. The constants in Eq. (16) are real and are defined by $P=(\hbar / i m) p_{S X}^{x}$,

$$
\begin{aligned}
& B=\frac{\hbar}{m^{2}} \sum_{i}^{\Gamma_{15}} p_{S i}^{y} p_{i X}^{z}\left(\omega_{X i}^{-1}+\omega_{S i}^{-1}\right), \\
& D=\frac{e}{m^{2}} \sum_{i}^{\Gamma_{15}} p_{S i}^{y} p_{i X}^{z}\left(\omega_{X i}^{-2}-\omega_{S i}^{-2}\right) .
\end{aligned}
$$

Since we are interested in optical anisotropy, it is helpful to rewrite Eq. (16) in terms of the rotated coordinates $x^{\prime}=$ $(x+y) / \sqrt{2}, y^{\prime}=(-x+y) / \sqrt{2}$, and $z^{\prime}=z$ :

$$
\begin{aligned}
\frac{\hbar}{i m}\left\langle S|\hat{\mathbf{p}}| X^{\prime}\right\rangle & =\hat{\mathbf{x}}^{\prime}\left(P+D E_{z}-i B k_{z}\right)+\hat{\mathbf{z}}\left(D E_{x^{\prime}}-i B k_{x^{\prime}}\right), \\
\frac{\hbar}{i m}\left\langle S|\hat{\mathbf{p}}| Y^{\prime}\right\rangle & =\hat{\mathbf{y}}^{\prime}\left(P-D E_{z}+i B k_{z}\right)-\hat{\mathbf{z}}\left(D E_{y^{\prime}}-i B k_{y^{\prime}}\right), \\
\frac{\hbar}{i m}\langle S|\hat{\mathbf{p}}| Z\rangle & =\hat{\mathbf{z}} P+\hat{\mathbf{x}}^{\prime}\left(D E_{x^{\prime}}-i B k_{x^{\prime}}\right)-\hat{\mathbf{y}}^{\prime}\left(D E_{y^{\prime}}-i B k_{y^{\prime}}\right) .
\end{aligned}
$$

This shows clearly that, if $\mathbf{E} \|$ [001], the optical transition rate is not the same for light polarized along the $x^{\prime}$ and $y^{\prime}$ axes. To lowest order, the anisotropy is linear in $E$.

I turn now to the task of calculating the absorption spectrum to first order in $E$ [thus neglecting Eq. (11)] for the case E $\|$ [001]. Excitonic effects $[20,21]$ are ignored. The first step is to solve the eigenvalue problem $\hat{H} F=\epsilon F$, which is 
a set of coupled first-order differential equations:

$$
\frac{\partial}{\partial k_{z}} F=\frac{i}{e E}(\tilde{H}-\epsilon) F,
$$

in which $\tilde{H}=\hat{H}-U$ is a polynomial of the second order in $k_{z}$. The conduction band $S$ is decoupled (to second order), so the solution to Eq. (20) for this band may be found in standard textbooks [22, p. 76] [23, § 7.8]:

$$
\begin{gathered}
F_{c}=(2 \pi e E)^{-1 / 2} \exp \left\{i \left[\left(A_{c x} k_{x}^{2}+A_{c y} k_{y}^{2}-\epsilon_{c}\right) k_{z}\right.\right. \\
\left.\left.+\frac{1}{3} A_{c z} k_{z}^{3}\right] / e E\right\} .
\end{gathered}
$$

Here $A_{c i}=\hbar^{2} / 2 m_{c i}$, where $m_{c i}$ is the effective mass in the $i$ direction, and $\epsilon_{c}$ is the energy eigenvalue.

For the degenerate valence band, Eq. (20) cannot be solved analytically as it stands. To obtain an analytical solution, one must invoke the "diagonal approximation" in which all off-diagonal matrix elements of $\tilde{H}$ are neglected. Such an approximation obviously cannot yield the correct energy and wave function for the strongly mixed valence states. However, due to the sum rule for oscillator strengths, the diagonal approximation provides a reasonably accurate description of the absorption spectrum $[3,24]$. This approximation is therefore adopted in what follows.

If the diagonal approximation is to give reasonable results, all off-diagonal terms should vanish at $\mathbf{k}=\mathbf{0}$. This is indeed the case for $\tilde{H}$ (up to first order in $E$ ), but, for zinc-blende crystals under stress-free boundary conditions, there is an additional contribution to the Hamiltonian from the piezoelectric effect. In this effect, the field $\mathbf{E}=\hat{\mathbf{z}} E$ generates a shear strain $\varepsilon_{x y}=\frac{1}{2} d_{14} E[25$, p. 116], which in turn generates a valence-band mixing of the form $\left\langle X\left|H_{\varepsilon}\right| Y\right\rangle=\sqrt{3} d \varepsilon_{x y}$ [26, p. 310], where $d_{14}$ is the piezoelectric coefficient and $d$ is a deformation potential. To diagonalize $H_{\varepsilon}$, one must use the basis $\left\{\left|X^{\prime}\right\rangle,\left|Y^{\prime}\right\rangle,|Z\rangle\right\}$.

Under the diagonal approximation, the valence-band wave functions $F_{v}$ are the same as Eq. (21), except one replaces $m_{c i}$ with $-m_{v i}$ and $\epsilon_{c}$ with $\epsilon_{v}+\epsilon_{g}$, where $\epsilon_{g}$ is the energy gap. The next step is to calculate the interband matrix elements of $\hat{\mathbf{p}}$, which may be expressed in terms of $P, B, D$, and the overlap integrals [23,27]

$$
\begin{gathered}
\left\langle F_{c} \mid F_{v}\right\rangle=\Theta^{-1} \operatorname{Ai}(x), \\
\left\langle F_{c}\left|k_{z}\right| F_{v}\right\rangle=i \kappa \Theta^{-1} \operatorname{Ai}^{\prime}(x) .
\end{gathered}
$$

Here $\operatorname{Ai}(x)=\pi^{-1} \int_{0}^{\infty} \cos \left(\frac{1}{3} u^{3}+u x\right) d u$ is the Airy function [28], $x=\left(\hbar^{2} k_{x}^{2} / 2 \mu_{x}+\hbar^{2} k_{y}^{2} / 2 \mu_{y}+\epsilon_{g}+\epsilon_{v}-\right.$ $\left.\epsilon_{c}\right) / \Theta, \quad \kappa=\left(2 \mu_{z} e E / \hbar^{2}\right)^{1 / 3}, \quad \Theta=\hbar^{2} \kappa^{2} / 2 \mu_{z}, \quad$ and $\mu_{i}^{-1}=m_{c i}^{-1}+m_{v i}^{-1}$.

The absorption coefficient $\alpha$ may then be calculated following the textbook treatment of the Franz-Keldysh effect $[23, \S 7.8]$, with the result

$$
\begin{aligned}
& \alpha=R \Theta^{1 / 2} \pi\left\{(1 \pm 2 D E / P)\left[\mathrm{Ai}^{2}\left(\beta_{ \pm}\right)-\beta_{ \pm} \mathrm{Ai}^{2}\left(\beta_{ \pm}\right)\right]\right. \\
&\left.\mp(B \kappa / P) \mathrm{Ai}^{2}\left(\beta_{ \pm}\right)\right\} .
\end{aligned}
$$

Here the upper (lower) sign is for light polarized along the $x^{\prime}\left(y^{\prime}\right)$ axis, and $\beta_{ \pm}=\left(\epsilon_{g} \mp \delta-\hbar \omega\right) / \Theta$, where $\delta=\frac{\sqrt{3}}{2} d d_{14} E$ is the piezoelectric splitting and $\hbar \omega$ is the photon energy. $R$ is given by

$$
R=\frac{e^{2} P^{2}}{2 \pi \varepsilon_{0} \hbar^{2} \omega n c}\left(\frac{2 \mu}{\hbar^{2}}\right)^{3 / 2},
$$

where $\mu=\left(\mu_{x} \mu_{y} \mu_{z}\right)^{1 / 3}, \varepsilon_{0}$ is the permittivity of free space, $n$ is the refractive index, and $c$ is the speed of light. The valence-band masses are $m / m_{v x^{\prime}}=\gamma_{1}+\gamma_{2} \pm 3 \gamma_{3}$, $m / m_{v y^{\prime}}=\gamma_{1}+\gamma_{2} \mp 3 \gamma_{3}$, and $m / m_{v z}=\gamma_{1}-2 \gamma_{2}$, where $\gamma_{i}$ is a Luttinger parameter.

Since Eq. (24) is valid only to first order in $E$, it is legitimate to perform a first-order Taylor series expansion of $\operatorname{Ai}\left(\beta_{ \pm}\right)$with respect to the parameter $\delta$. Noting that $(d / d x)\left[x \mathrm{Ai}^{2}(x)-\mathrm{Ai}^{\prime 2}(x)\right]=\mathrm{Ai}^{2}(x)$ [23], one obtains

$$
\begin{aligned}
\alpha=R \Theta^{1 / 2} \pi\left\{(1 \pm 2 D E / P)\left[\mathrm{Ai}^{\prime 2}(\beta)-\beta \mathrm{Ai}^{2}(\beta)\right]\right. \\
\left. \pm(\delta / \Theta-B \kappa / P) \mathrm{Ai}^{2}(\beta)\right\},
\end{aligned}
$$

in which $\beta=\left(\epsilon_{g}-\hbar \omega\right) / \Theta$. This shows that the parameters $\delta$ and $B$, although of different physical origin, give rise to the same frequency dependence of $\alpha$.

The anisotropy in the absorption spectrum is defined by $\rho=\left(\alpha_{x^{\prime}}-\alpha_{y^{\prime}}\right) /\left(\alpha_{x^{\prime}}+\alpha_{y^{\prime}}\right)$. Since the absorption is significant primarily for $\hbar \omega>\epsilon_{g}$, one can simplify the above results by using the asymptotic expression $\mathrm{Ai}(-x) \sim \pi^{-1 / 2} x^{-1 / 4} \sin \left(\frac{2}{3} x^{3 / 2}+\frac{\pi}{4}\right)$ [28], which is valid in the limit $x \rightarrow \infty$. Hence, when $\hbar \omega-\epsilon_{g} \gg \Theta$,

$$
\begin{aligned}
\rho=E & {\left[2 D / P+\left(\frac{\sqrt{3}}{2} d d_{14}-e B / P\right)\left(\hbar \omega-\epsilon_{g}\right)^{-1}\right.} \\
& \left.\times \sin ^{2}\left(\frac{2}{3}|\beta|^{3 / 2}+\frac{\pi}{4}\right)\right] .
\end{aligned}
$$

The absorption coefficient and anisotropy for GaAs are plotted versus frequency in Fig. 1. In this calculation, the following experimental parameters were used: $2 m P^{2}$, $\hbar^{2}=28.9 \mathrm{eV}, \quad \epsilon_{g}=1.424 \mathrm{eV}, \gamma_{1}=7.10, \quad \gamma_{2}=2.02$, $\gamma_{3}=2.91, \quad m_{c}=0.067 m, \quad d=-4.55 \mathrm{eV}, \quad d_{14}=$ $-2.69 \times 10^{-12} \mathrm{~m} \mathrm{~V}^{-1}$, and $n=3.299$. In addition, the values $B / P=0.163 \AA$ and $D / P=2.69 \times 10^{-12} \mathrm{~m} \mathrm{~V}^{-1}$

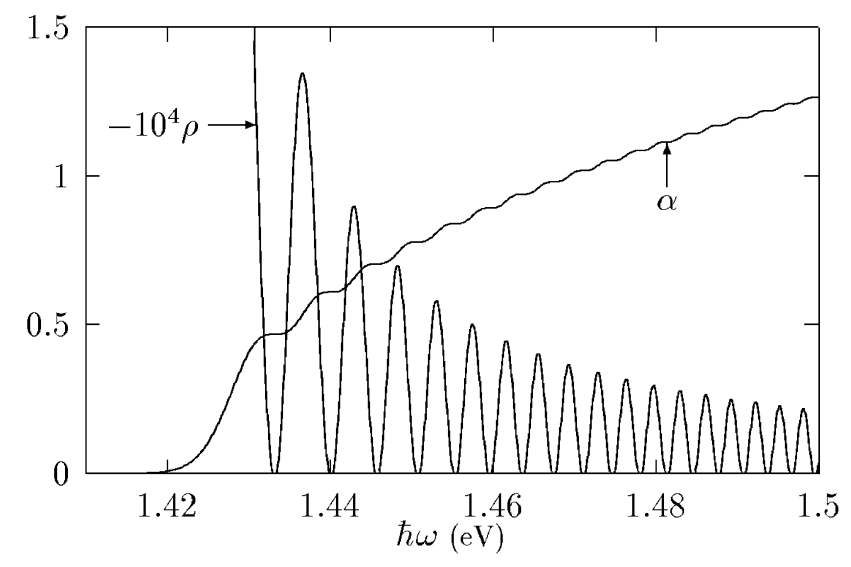

FIG. 1. Absorption coefficient $\alpha\left(\mu \mathrm{m}^{-1}\right)$ and anisotropy $\rho$ calculated from Eq. (24) with $E=3000 \mathrm{~V} / \mathrm{cm}$. 
were calculated from the empirical pseudopotentials in Ref. [29]. (The fact that $d_{14}=-D / P$ is coincidental.)

The absorption spectrum in Fig. 1 shows the expected Franz-Keldysh oscillations; no difference between $\alpha_{x^{\prime}}$ and $\alpha_{y^{\prime}}$ is visible at this scale. These oscillations dominate the anisotropy spectrum, because $\rho$ in Eq. (27) is proportional to $(d \alpha / d \omega) / \alpha$. The neglect of such oscillations in Ref. [1] is therefore unjustified.

In Eq. (27), the ratio of the contribution from $D$ to that from $\delta$ and $B$ (which are of comparable magnitude) is $\left(\hbar \omega-\epsilon_{g}\right) / \Delta \epsilon$, where $\Delta \epsilon$ is on the order of several electron volts. The contribution from $D$ is therefore negligible in the vicinity of the band gap.

Spin-orbit coupling does not qualitatively alter the results found above. If one uses the basis kets $\left|j, m_{j}\right\rangle$ given in Ref. [30], the strain Hamiltonian for the $\Gamma_{8}$ band is $\left\langle\frac{3}{2}, \pm \frac{3}{2}\left|H_{\varepsilon}\right| \frac{3}{2}, \mp \frac{1}{2}\right\rangle=i \delta / \sqrt{3}$. This is diagonalized by the kets $\frac{1}{\sqrt{2}}\left|\frac{3}{2}, \pm \frac{3}{2}\right\rangle-\frac{i}{\sqrt{2}}\left|\frac{3}{2}, \mp \frac{1}{2}\right\rangle$ and $\frac{1}{\sqrt{2}}\left|\frac{3}{2}, \pm \frac{3}{2}\right\rangle+\frac{i}{\sqrt{2}}\left|\frac{3}{2}, \mp \frac{1}{2}\right\rangle$. The absorption coefficient is thus

$$
\alpha_{\Gamma_{8}}=\frac{1}{3}\left(1+\frac{\sqrt{3}}{2}\right) \alpha\left(\beta_{ \pm}^{\prime}\right)+\frac{1}{3}\left(1-\frac{\sqrt{3}}{2}\right) \alpha\left(\beta_{\mp}^{\prime}\right),
$$

in which $\alpha$ is the function defined in Eq. (24), $\beta_{ \pm}^{\prime}=$ $\left(\epsilon_{g} \mp \delta / \sqrt{3}-\hbar \omega\right) / \Theta$, and the valence-band masses are $m / m_{v x^{\prime}}=\gamma_{1} \pm \sqrt{3} \gamma_{3}, m / m_{v y^{\prime}}=\gamma_{1} \mp \sqrt{3} \gamma_{3}$, and $m / m_{v z}=\gamma_{1}$. The asymptotic expression for $\rho$ is the same as (27), except the piezoelectric contribution is reduced by a factor of $\frac{1}{2}$.

For the $\Gamma_{7}$ band, the strain Hamiltonian is zero, so the basis kets are $\left|\frac{1}{2}, \pm \frac{1}{2}\right\rangle$. The absorption coefficient is thus

$$
\alpha_{\Gamma_{7}}=\frac{1}{3} \alpha\left(\beta_{\Delta}\right)
$$

in which $\beta_{\Delta}=\left(\epsilon_{g}+\Delta-\hbar \omega\right) / \Theta$, where $\Delta$ is the spinorbit splitting. The valence-band mass is $m / \gamma_{1}$ in all directions. The asymptotic expression for $\rho$ is the same as (27), except the piezoelectric contribution is omitted. Equation (29) is consistent with the $\Gamma_{7}$ Pockels-effect model of Aronov and Pikus [27].

The present theory is, however, qualitatively different from more recent publications. Khurgin and Voisin [1] and Krebs and Voisin [3] have proposed that the LuttingerKohn Hamiltonian should include a linear-field term that mixes the $X$ and $Y$ valence states. Such a term would be similar to the piezoelectric mixing $\delta$ considered here, but its physical origin would be entirely different, since it would arise from polarization of the valence electrons rather than displacement of the lattice. However, Eq. (7) shows clearly that no such term exists in the LuttingerKohn Hamiltonian. Linear-field effects arising from elec- tronic polarization are described instead by the terms $B$ and $D$ in the momentum matrix.

Zhu and Chang [15] proposed a Hamiltonian that contains both the $\mathbf{k} \cdot \mathbf{p}$ interaction (2) and the dipole coupling (7). This is incorrect, since the transformation $e^{S}$ that generates (7) also eliminates (2).

This work was supported by HKUST Grant No. DAG99/00.SC27.

[1] J. B. Khurgin and P. Voisin, Phys. Rev. Lett. 81, 3777 (1998).

[2] S. H. Kwok, H. T. Grahn, K. Ploog, and R. Merlin, Phys. Rev. Lett. 69, 973 (1992).

[3] O. Krebs and P. Voisin, Phys. Rev. Lett. 77, 1829 (1996).

[4] O. Krebs et al., Phys. Rev. Lett. 80, 5770 (1998).

[5] R. Magri and S. Ossicini, Phys. Rev. B 58, R1742 (1998).

[6] E. L. Ivchenko, A. A. Toropov, and P. Voisin, Phys. Solid State 40, 1748 (1998).

[7] B. A. Foreman, Phys. Rev. Lett. 82, 1339 (1999).

[8] L. V. Keldysh, Soviet Phys. JETP 7, 788 (1958).

[9] W. Franz, Z. Naturforsch. 13A, 484 (1958).

[10] J. Callaway, Phys. Rev. 130, 549 (1963).

[11] E. N. Adams II, J. Chem. Phys. 21, 2013 (1953).

[12] E. I. Blount, in Solid State Physics, edited by F. Seitz and D. Turnbull (Academic, New York, 1962), Vol. 13, pp. 305-373.

[13] G. H. Wannier, Phys. Rev. 117, 432 (1960).

[14] J. M. Luttinger and W. Kohn, Phys. Rev. 97, 869 (1955).

[15] B.-F. Zhu and Y.-C. Chang, Phys. Rev. B 50, 11932 (1994).

[16] The symbol $|n\rangle$, unlike $|n \mathbf{k}\rangle$, has no independent meaning (i.e., $|n\rangle$ is not a Dirac ket vector).

[17] G. Dresselhaus, Phys. Rev. 100, 580 (1955).

[18] This technique was used in the Bloch representation by E. O. Kane, J. Phys. Chem. Solids 12, 181 (1959).

[19] R. H. Parmenter, Phys. Rev. 100, 573 (1955).

[20] J. D. Dow and D. Redfield, Phys. Rev. B 1, 3358 (1970).

[21] D. F. Blossey, Phys. Rev. B 2, 3976 (1970).

[22] L.D. Landau and E.M. Lifshitz, Quantum Mechanics (Pergamon, Oxford, 1977), 3rd ed.

[23] A.I. Anselm, Introduction to Semiconductor Theory (Prentice-Hall, Englewood Cliffs, NJ, 1981).

[24] Y.-C. Chang and J. N. Schulman, Phys. Rev. B 31, 2069 (1985).

[25] J.F. Nye, Physical Properties of Crystals (Oxford, New York, 1985).

[26] G. L. Bir and G. E. Pikus, Symmetry and Strain-Induced Effects in Semiconductors (Wiley, New York, 1974).

[27] A. G. Aronov and G. E. Pikus, Sov. Phys. Solid State 10, 648 (1968).

[28] M. Abramowitz and I. A. Stegun, Handbook of Mathematical Functions (Dover, New York, 1964).

[29] K. A. Mäder and A. Zunger, Phys. Rev. B 50, 17393 (1994).

[30] B. A. Foreman, Phys. Rev. B 48, 4964 (1993). 